\title{
An investigation on a new sliding cutting strategy for Carbon Fiber-Reinforced Polymers
}

Qingxun Meng ( $\sim$ mqx@sau.edu.cn )

Shenyang Aerospace University

\section{Zheng Huang}

Jin Cai

Xin Pan

Hui Zhang

\section{Research Article}

Keywords: CFRP, sliding cutting, cutting strategy, orthogonal cutting

Posted Date: March 3rd, 2022

DOI: https://doi.org/10.21203/rs.3.rs-1399868/v1

License: (1) This work is licensed under a Creative Commons Attribution 4.0 International License. Read Full License 


\section{Abstract}

The current cutting strategies for carbon fiber reinforced polymer (CFRP) inherit essentially from metal cutting. The frequently occurred damages such as burrs, cracks and delamination suggest that the existed strategies may not be the best choice for CFRP cutting. This study proposed a sliding cutting strategy especially for CFRP. The novelty is adding an extra lateral velocity on cutting edge to act sliding motion between fiber-matrix and the cutting edge. Then the stress in the materials in front of cutting tip will become more concentrated, which will help to break fibers in desired positions. Thus, the machining quality can be improved. The sliding cutting process was analyzed and experiments were conducted to examine the proposed strategy. Two types of cutting tools that offered sliding and orthogonal cutting were designed. Four factors, namely machined surface roughness, subsurface damages, forces and tool wear, were obtained to evaluate the cutting quality. Results show that sliding cutting has more advantages than orthogonal cutting in CFRP machining.

\section{Introduction}

Carbon fiber-reinforced polymer (CFRP) has been widely used in aerospace industry due to the lightweight, high strength and corrosion resistant properties. Its application is changing from non-bearing structures to bearing structures on airplane in the past decades [1]. Before assembling into products, machining works such as drilling or milling are necessary for CFRP parts to satisfy mechanical and accuracy requirements. With the increasing expectation of load bearing strength, higher CFRP machining quality is required. Damages are getting unacceptable during drilling and milling since they may increase potential failure risks of CFRP structures.

In order to reduce CFRP machining damages, researchers studied CFRP orthogonal and oblique cutting, which are the basic forms of drilling, reaming and milling. The most concerned values that used to evaluate cutting quality are machined surface roughness and subsurface damage. The forces during machining are also regarded as indicators to estimate machining state. Generally, the smaller and the less fluctuate forces mean the cutting process is more stable and energy-saving. Wang et al [2] and An et al [3] designed experiments respectively to investigate orthogonal cutting of unidirectional CFRP. Machined surface roughness, subsurface damage and cutting forces were measured using fiber orientation as variables. It was found the fiber orientation is the key factor that affects cutting process. With the developing of numerical techniques, simulation models in micro scale [4]-[6], macro scale [7] [8] and multi-scale [9][10] have been built to study the so called cutting mechanism. Similar results to Wang's work were found that fiber orientation controls machined surface roughness, subsurface damage and cutting forces. Research results also showed that cutting depth [11][12], cutting speed [13] and rake angle [14] are the relevant parameters that affect machining quality. Since the variation of fiber orientation is unchangeable during machining [15], attempts associate with machining parameters optimizing were made to reduce machined surface roughness, subsurface damages and cutting forces. Parameters optimizing is effective to some extent, but it is meaningful only when cutting CFRP with proper strategy. 
Different CFRP cutting strategies have been proposed by researchers in literature. One of the most typical strategies is changing the continuous cutting to the discrete cutting. Specifically, long cutting edges are interrupted to create a number of short rhomboid cutting edges [16]. In this way, a certain amount of fibers and matrix to be removed can be cut little by little instead of being cut at one time. Cutting load is dispersed and thus damages and tool wear will be reduced. The second CFRP cutting strategy is used to eliminate burrs during milling. Cutting edges on a milling tool are made herringbone [17]. Then the fibers and matrix on each margin of a laminate are pushed toward inner layers side before being cut. Fibers outof-plane deformation is reduced hence burrs can be eliminated during milling. The third CFRP cutting strategy is reverse cutting. The cutting direction is changed from downward to upward by setting reverse cutting edges on a drill [18][19]. As the drill rotating, the reverse cutting edges act forces on the fibers around hole-exit side in a direction opposite to feed. By this means, though burrs cannot be prevented, they can be removed immediately once occurring. The fourth CFRP cutting strategy is step-control cutting, which means cutting process in a single tool is divided into at least two steps. Each step executes specific purpose. V-shaped cutting edges and diameter ratio controlled tool are presented in Ref. [20] [21] and Ref. [22] respectively. The step-control strategy focuses on cutting hole center materials in the first step to avoid the effects of thrust force. Then the remained materials around hole-exit are cut more carefully in the next step.

In narrow sense, CFRP cutting strategy refers to how cutting edges with specific geometry move, approach and cut fiber-matrix. At present, almost all the CFRP cutting strategies inherit essentially from metal cutting. The basic cutting forms are still orthogonal and oblique cutting, seen from a point on cutting edge. Metal cutting has been developed for more than a hundred years. It is defined as a forming process with purposeful fracture of the layer being removed [23]. During metal cutting, the maximum combined stress does not necessary act at the cutting edge. In most time, rake face plays a key role to push the front materials conducting plastic deformation. Therefore, the wear may occur at a certain distance from the cutting edge. This allows maintaining the accuracy of machining over the entire time of tool life [23]. According to the research results in literature [24], however, CFRP cutting rely almost entirely on cutting edge instead of rake face to break fibers and matrix. In this way, cutting edge gets wear fast and machining quality is sensitive to tool wear. Moreover, CFRP is generally considered as brittle material and there is barely plastic deformation during cutting. Therefore, materials properties determine that CFRP cutting is greatly different from metal cutting. These suggest that strategies inherited from metal cutting may not be the best choice for CFRP cutting.

This study is trying to establish a new cutting strategy. The novelty lies in extra tangential velocity is provided for an arbitrary point on cutting edge. Then the interaction between cutting edge and fibers will be changed. Four factors, namely machined surface roughness, subsurface damage, forces and tool wear, are used to evaluate the new CFRP cutting strategy.

\section{Definition And Analysis Of Cfrp Sliding Cutting Strategy}


Tool geometry is a key factor that determines the CFRP cutting strategy. In most situations, the geometry varies along cutting edges. Therefore, the strategy is described using a segment on cutting edge. Fig. 1a shows a schematic of typical CFRP cutting. Assume AB is a segment of a cutting edge and a point $i$ is on AB. A Cartesian coordinate is built with the origin point $o$ overlaps with point $i$. Axis $x$ is parallel to assumed primary motion $\left(v_{c}\right)$, while axis $z$ is opposite to feed motion $\left(v_{f}\right)$. Axis $y$ is determined by axis $x$ and $z$ according to the right-hand rule.

During cutting of CFRP laminates, the fibers direction varies in plain xOz. Fiber orientation angle (FOA), which is a key factor that affects cutting quality, is simply defined as an angle between fiber and primary motion $v_{c}$ According to the spatial relationship between fibers and cutting edge, FOA varies from $0^{\circ}$ to $180^{\circ}$ [15]. Inclination angle $\lambda$ is an angle between cutting edge $A B$ and tool reference plane (measured in plane $x o y$ ). When $\lambda=0^{\circ}$, the cutting process is characterized as orthogonal cutting. The force acting on the fiber by the cutting edge $A B$ is parallel to $v_{O}$ as shown in Fig. $1 \mathrm{~b}$. When $\lambda>0^{\circ}$, the cutting process is oblique cutting. In this situation, the cutting edge offers normal force $F_{n}$ and friction force $F_{s}$ to the fiber, as shown in Fig. 1 c. $F_{t}$ is component force of $F_{n}$ in the direction that parallel to AB. $F_{t}$ and $F_{n}$ is dependent on the inclination angle $\lambda$. During oblique cutting, there exists a specific $\lambda$ that makes the value of $F_{t}$ equals to $F_{n}$. In this situation, theoretically, the fibers will go along the direction of $v_{c}$ Otherwise, the fibers will deviate from $v_{c}$ direction and slide on cutting edge due to subjecting to unbalanced force. Therefore, oblique cutting is also taken as sliding cutting in literature [25]. This sliding, however, is considered as passive sliding cutting, since the cutting edge keeps static in lateral direction. The passive sliding cutting has been reported in agricultural cutting tool design. In this study, an active sliding cutting for CFRP is presented. The "active" means that a point on cutting edge moves not only in primary $\left(v_{c}\right)$, but also moves along the tangential direction of the point on the cutting edge in a speed of $v_{S^{\prime}}$ as shown in Fig. 2.

In literature, fibers and matrix breaking mechanism during CFRP machining was study by investigating orthogonal cutting [26]. According to the research results, extensive shearing occurs in the area in front of cutting tip. In shearing zone, as shown in Fig. 2a, cutting tip pushes fibers and matrix to form chips while subsurface materials still hold them to stay. Thus, the fibers in cutting area experience compressing, bending and tension before being cut [6][27]. This causes the stress concentration points in fibers scattered around nominal cutting surface. Therefore, fibers may break in the positions on or below the nominal cutting surface in the shearing zone.

Compared to the typical orthogonal cutting (or oblique cutting), sliding cutting tends to narrow the shearing zone. Since there exists high speed lateral relative motion between cutting edge and fibers, shearing stress is focalized near the contacting point of cutting tip and fiber, as shown in Fig. 2b. Except for compressing, the cutting edge mainly offers intensity grinding and shearing to the fibers. Hence, the fibers tend to be broken in front of cutting edge before experiencing forward bending. Therefore, less deviation exists between fibers breaking positions and nominal cutting surface. The energy consumption 
during cutting is then reduced. As a result, the first potential advantage of sliding cutting is it improves machined surface quality and the second one is it reduces cutting force.

For a single fiber during sliding cutting, the forces acted on it are different from that in orthogonal and oblique cutting (Fig. 1b and Fig. 1c). The forces are dependent on sliding cutting direction and inclination angle. Three situations of sliding cutting are listed in Fig. 3 and the forces are analyzed. The angle $\left(\lambda_{s}\right)$ between $v_{c}$ and $v_{s}$ is used to characterize the situations. The cutting edge acts friction force $\left(F_{s}\right)$ and normal force $\left(F_{n}\right)$ to the fiber. The force $\left(F_{c}\right)$ in primary motion direction is defined as chopping force, while the force $\left(F_{t}\right)$ parallel to cutting edge is defined as sliding cutting force. In the first situation (Fig. $3 a), \lambda_{s}$ is acute angle and the direction of $F_{t}$ and $F_{s}$ is opposite to each other. In the third situation (Fig. 3c), however, $\lambda_{s}$ is obtuse angle and the direction of $F_{t}$ and $F_{s}$ is the same. There is no sliding cutting force in the second situation, as shown in Fig. $3 \mathrm{~b}$.

This means that the fibers motion can be determined by adjusting friction force $F_{s}$ and inclination angle $\lambda$. Then the third potential advantage of sliding cutting comes that the subsurface deformation can be more likely controlled. According to the previous analysis, the tool wear of sliding cutting remains unclear.

\section{Experimental Methodology And Set Up}

In order to verify the presented conceptions and analysis, sliding cutting tools were designed and experiments were conducted. Three-axis milling machine was used to carry out the experiments. Spindle rotation was utilized to offer sliding motion, while the drive in 3 axes were used to offer primary and feed motion. Two types of customized cutting tools were made, as shown in Fig. 4a. The first type was sliding cutting tool. For experimental purpose, the cutting edge was made in a circle. It conducts sliding cutting when spindle rotates. The rake and flank angle of the tool were made $30^{\circ}$ and $10^{\circ}$ respectively. The second type was orthogonal cutting tool. The rake and flank angle were the same to the sliding cutting tool. Orthogonal cutting was taken as control group and the inclination angle $(\lambda)$ is $0^{\circ}$. The tools in experiments were made sharp (small cutting wedge angle) to generate more concentrated stress in fibers, though it may increase the risk of wear and flanking. All the cutting tools were made of cemented carbide.

CFRP (T300/7901) laminates were offered by Shenyang Aircraft Industry (Group) Co. LTD. The stacking sequence was $\left[0^{\circ}\right]_{20}$. Test-pieces were cut to a configuration of $60 \mathrm{~mm} \times 60 \mathrm{~mm} \times 4 \mathrm{~mm}$ from larger laminates. FOAs were set to $0^{\circ}, 45^{\circ}$ and $90^{\circ}$ by adjusting the location of the test-pieces, as shown in Fig. $4 \mathrm{~b}$. The cutting depth for both sliding and orthogonal cutting were adjusted to $0.04 \mathrm{~mm}$. The spindle speed and feed rate were set $1000 \mathrm{r} / \mathrm{min}$ and $1.2 \mathrm{~m} / \mathrm{min}$ respectively.

The experimental set up is shown in Fig. 5. The cutting tools were installed on a milling machine. The CFRP test-pieces were fixed by a clamp. Then the clamp was fastened on a dynamometer (Kistler 9257A) in order to measure cutting, thrust and lateral forces. The surface to be machined was adjusted to keep horizontal. Then a Kistler 5073 charge amplifier was connected to the dynamometer. The forces signals were amplified and transmitted to a HBM GEN2i type data acquisition. The sampling frequency was set 
to $1 \mathrm{kHz}$ for each channel. After sliding and orthogonal cutting, the machined surface and subsurface were examined using VHX-1000 type digital 3D microscope.

Extra experiments were conducted to measure tool wear for sliding cutting and orthogonal cutting. Two new tools for each type were prepared. The cutting edge rounding radius of the new tools was measured using the fitting function of the digital microscope. Then both tools were used to cut UD-CFRP test-pieces with nominal cutting depth of $0.04 \mathrm{~mm}$. After that, the cutting edge rounding radius was measured again.

\section{Results And Discussion}

\subsection{Machined surface quality}

The machined surface and its roughness of each FOA was examined using microscope, as shown in Fig. 6. During orthogonal cutting, fibers and matrix are cut and separated by cutting wedge. Then cutting chips move on rake face and the materials remained on machined surface are pressed and ground by flank face. The grinding interaction between flank face and machined surface goes toward cutting direction. In sliding cutting, however, the grinding interaction goes not only in cutting direction but also in lateral direction. This causes the machined surface quality during sliding cutting to become lower than that of orthogonal cutting when FOA is $0^{\circ}$. In this situation, the sliding action does not help to cut fibers since the fibers are parallel to cutting direction. The fibers on machined surface undergo vertical grinding effects offered by flank face. Breaking and peeling occur frequently on the machined surface and hence the quality is slightly reduced, as shown in Fig. 6 a.

When FOA is $45^{\circ}$, the advantage of sliding cutting is revealed thoroughly. Fig. $6 \mathrm{~b}$ shows that the machined surface quality of sliding cutting is higher than orthogonal cutting. After sliding cutting, fibers are cut uniformly right in front of the cutting edge and the fractures are regular and neat. There is no obvious irrelevant breaking and peeling. The matrix in machined surface is also much smoother than orthogonal cutting. The removal of matrix is highly related to fibers breaking. During orthogonal cutting, fibers are crushed by cutting edge. The breaking position scattered around nominal machined surface [24]. At the same time, micro-cracks occur extensively in the surrounding matrix. Hence, the fracture path during cutting chips formation tends to go randomly. This leads to a rough machined surface after orthogonal cutting. When the fibers are broken in desired position by sliding motion of the cutting edge, the fracture in matrix will go in a relatively fixed path. These explain the difference of machined surface quality between the two machining methods. The previous analysis that sliding cutting generates higher concentrating stress in fibers can also be proved.

When $\mathrm{FOA}$ is $90^{\circ}$, the machined surface quality of sliding cutting still remains higher than orthogonal cutting. The reason is similar as the situation when $\mathrm{FOA}=45^{\circ}$. The roughness of machined surface (both of sliding and orthogonal cutting), however, is lower than that of $F O A=45^{\circ}$. This is because the back support of fibers becomes weaker and weaker as FOA increases from $45^{\circ}$ to $90^{\circ}$, causing fibers experience larger deformation before being cut. The variation of machined surface roughness is shown in 
Fig. $6 \mathrm{~d}$. The roughness of sliding cutting is much lower than that of orthogonal cutting, except for $\mathrm{FOA}=0^{\circ}$.

\subsection{Subsurface damages}

Subsurface is generally considered as a region that beneath and near to the machined surface. Subsurface damage is an important indicator that reflects machining quality. In some cases, a good machined surface quality does not necessarily mean good machining quality because damages may be covered in subsurface. In order to check the damages, small cubes have been taken from the test-pieces by cutting and polishing, as show in the schematic figures in Fig. 7 to Fig. 9. Side and back views (the plane $\left.P_{o}, P_{S}, P_{o 1,2}, P_{S 1,2}\right)$ are selected to reveal the morphology of the subsurface.

When $\mathrm{FOA}=0^{\circ}$, there is no subsurface damages found in orthogonal cutting, as shown in Fig. 7a. Only side view (plane $P_{o}$ and $P_{S}$ ) is given because damages or deformation cannot be seen clearly in back view. The fibers and matrix are separated and lifted by rake face [24]. Therefore, the deformation in cutting area does not transmit to the subsurface. There is also barely subsurface damage found in sliding cutting. The only problem is a few fibers are left as burrs after ground by flank face, as shown in Fig. 7b. The damages are not found extend to the subsurface. The burrs formation reason has been discussed when analyzing machined surface. Overall, the subsurface quality during orthogonal and sliding cutting is good enough since there are no large cracks and debonding in the fibers and matrix.

Subsurface damage still keeps relatively low level when FOA $=45^{\circ}$. Seen from the back views (plane $P_{o 1}$ in Fig. 8a), the yellow contour line shows slightly fluctuation in orthogonal cutting. This means undesirable fibers breaking occurred during cutting. Despite this, there are no large cracks found in the subsurface. The contour line of sliding cutting, however, is much smoother, as shown in plane $P_{s 1}$ in Fig. 8c. It indicates that the fibers are cut right in front of cutting edge. This is a strong proof of the analysis in Section 2 that more concentrating stress generated in fibers during sliding cutting. A peculiar phenomenon, however, is found that the fibers experience lateral deformation after being cut by sliding cutting edge (as shown in Fig. 8c). The deformation trend is in the same direction with sliding cutting velocity. This means the fibers undergo lateral force acted by sliding. Further analysis will be presented when $\mathrm{FOA}=90^{\circ}$ as the trend becomes more obvious. There is no such phenomenon found in orthogonal cutting (as shown in Fig. 8a).

When checking from the side view of orthogonal cutting (Plane $P_{o 2}$ in Fig. 8b), visible subsurface damage can be clearly found. Fibers breaking positions are becoming scattered. Some fibers are broken in the position that beneath the machined surface, causing fractures in subsurface. The reason has reported in literature [24] and can be explained according to the analysis in Fig. 2. When checking the subsurface after sliding cutting, damages are barely found, as shown in Fig. 8b. Though experiencing lateral deformation, the subsurface quality is much better than that in orthogonal cutting. The reason is same to the discussion when checking the back views. 
Subsurface damage becomes severer when $\mathrm{FOA}=90^{\circ}$ during orthogonal cutting. Seen from both back view (Fig. 9 a) and side view (Fig. 9 b), the yellow contour lines of subsurface sections fluctuate dramatically. The fibers on the sections tend to break deviating from the machined surface. During sliding cutting, most fibers broken positions locate uniformly on machined surface. The subsurface quality is much higher than orthogonal cutting. These indicate that sliding cutting generates higher concentrating stress in fibers than orthogonal cutting. This is an overwhelming advantage of sliding cutting.

The fibers lateral deformation (in Plane $P_{s 1}$ ) phenomenon, which has been reported when $\mathrm{FOA}=45^{\circ}$, was found again. The deformation becomes larger as FOA increases from $45^{\circ}$ to $90^{\circ}$. Fig. $9 \mathrm{~d}$ shows the deformed fibers and matrix in Plane $P_{s 2}$ have been removed after polishing (the dark area encircled by the yellow dashed line). The reason of the deformation, according to the analysis and experimental results in this study, is the fibers are bended by the lateral forces during sliding cutting, as shown in Fig. 2 and Fig. 3. Though it seems sufficient to explain the phenomenon, a contradiction comes out. That is the fibers in Plane $P_{o 2}$ also undergo forces in perpendicular direction, but they keep straight instead of deformed after being cut. Orthogonal cutting is characterized as instantaneous cutting, which means fibers and the surrounded matrix contact a small section of cutting edge only when they are broken and separated. Then the cutting edge moves forward to conduct next cutting action. Sliding cutting, however, can be considered as continuous cutting. A long section of cutting edge slides to grind and cut fibers and the surrounded matrix. The interaction between sliding cutting edge and fiber-matrix is much intensive compared to orthogonal cutting. The contradiction can be explained, to some extent, by the instantaneous and continuous cutting. Another potential reason is cutting heat which is not monitored in the experiment in this study. Sliding cutting may generate more heat and as a result resin matrix is softened. This will also cause the difference of fibers deformation between sliding and orthogonal cutting.

Though obvious damages are not found in the subsurface shown in Fig. 9c, fibers lateral deformation is not expected during sliding cutting. According to the analysis in section 2, the forces acted on the fibers are dependent on the angle $\left(\lambda_{s}\right)$ between $\boldsymbol{v}_{c}$ and $\boldsymbol{v}_{\boldsymbol{s}}$ For the convenience of discussing, five fiber locations are selected as shown in Fig. 9c. The fibers bending trend experiences small-large-small from location 1 to 5 . At the same time, $\lambda_{s}$ changes from acute to obtuse angle. The largest fiber bending is in location 3 where $\lambda_{s}$ is $90^{\circ}$ and the lateral force reaches maximum value. The results are strong proof of the analysis in Fig. 3. Theoretically, based on the analysis and the experimental results, fibers lateral deformation can be reduced or even prevented by adjusting cutting tool geometry and cutting parameters.

\subsection{Forces during cutting}

Forces between cutting tool and test-pieces are key indicators that reflect the status during cutting [28]. Large forces generally mean large energy consumption and severe fluctuation forces indicate that the cutting process is experiencing unstable time. Fig. 10 shows the forces during sliding and orthogonal cutting. The forces in $x, y$ and $z$ axis are cutting force, lateral force and thrust force respectively. According to the curves in Fig. 10a, the average cutting force of orthogonal cutting increases as FOA 
varies from $0^{\circ}$ to $90^{\circ}$. Its growth rate keeps positive and the force fluctuation gets severer. The average cutting force of sliding cutting also increases in the situation, but the values are much lower than that of orthogonal cutting. Especially when FOA is $90^{\circ}$, the cutting force drops nearly $74 \%$. The growth rate of sliding cutting force is getting negative and the fluctuation tends to get smooth. This is a positive evidence of the analysis in section 2 that energy consumption reduced when cutting fibers since intensity grinding and shearing to the fibers are offered.

Fig. 10b shows thrust forces during sliding and orthogonal cutting. It can be seen that sliding cutting does not reduce thrust force greatly. The variation trend and the fluctuation of thrust force are also not changed. This is because both sliding and orthogonal cutting contact machined surface through tool flank face. The interaction does not change after adding lateral velocity on cutting edge. Fig. 10c shows the forces in lateral direction. The lateral forces signals were only detected during sliding cutting, which cause fibers lateral deformation in the subsurface (Fig. 8 and Fig. 9). Moreover, the variation trend of the lateral force is consistent the thrust force. The two phenomenon indicate that the lateral force is determined by the interaction of tool flank face and machined surface. There is no lateral force acted on the fibers during orthogonal cutting, since the cutting edge is perpendicular to resultant cutting motion.

The comparison results mean that sliding cutting is steadier and needs less energy than orthogonal cutting. The data variations offer a positive evidence that sliding cutting is a better choice for CFRP cutting.

\subsection{Tool wear}

Orthogonal and sliding cutting tools with the same rake and flank angles were used to examine tool wear. Test-pieces with $45^{\circ} \mathrm{FOA}$ were taken as cutting samples. The cutting length of orthogonal cutting tool was 12 meters $(60 \mathrm{~mm} \times 200$ times). The nominal cutting depth was $0.04 \mathrm{~mm}$ but the actual cutting depth was depend on the accuracy of milling machine. Therefore, the cutting length of sliding cutting was made 20 meters in order to eliminate the possible affection of mechanical precision.

Fig. 11a shows how the cutting tool wear was measured. Cutting edge rounding radius was used as wear indicator. The 3D features of cutting edge were measured by the digital microscope. Then a section that perpendicular to cutting edge on the checking point was taken. A circle was then drawn using the built-in software to fit the curve of cutting edge rounding. Fig. 11b and $\mathrm{c}$ are the measurements of the rounding radiuses of the orthogonal and sliding cutting respectively. As can be seen from the figures, the radius of the new tools is almost the same. After experiencing extra several meters cutting length, the cutting edge rounding radius sliding cutting tool is smaller than that of orthogonal cutting tool. It indicates that sliding cutting gets wear slower than orthogonal cutting.

This is an unexpected result. Based on previous experience, sliding cutting is considered easy to wear since continuous grinding exists during the whole cutting time. The final results, however, turn out to the opposite side that sliding cutting shows better wear resistance than orthogonal cutting. Though the analysis in section 2 is inadequate to explain the reason, it can be discussed according to the two 
following aspects. The first one is the cutting forces during sliding cutting is reduced significantly compared to orthogonal cutting. This means more energy has been saved and then tool wear becomes weaker. The second one is only a small part of cutting edge engaged during orthogonal cutting. It can be clearly seen in Fig. 11b around the checking point. During sliding cutting, however, the whole cutting edge take part in cutting. It means "cutting load" has been shared by the whole cutting edge. Tool wear speed is then reduced.

\section{Conclusions And Out Look}

This study proposed a new CFRP sliding cutting strategy. The cutting quality was compared with orthogonal cutting using four factors, namely machined surface roughness, subsurface damages, cutting forces and tool wear. According to the results, sliding cutting showed more advantages than orthogonal cutting. The proposed strategy is a potential method to improve CFRP machining quality after being adopted in tool design. Sliding cutting is able to generate more concentrating stress in fibers, which helps to break fibers in desired positions. The following findings will help to understand the advantage of CFRP sliding cutting strategy:

1) The unique feature of the CFRP sliding cutting presented this study was that lateral relative velocity, except for primary motion and feed, was offered between cutting edge and fiber-matrix. Extra energy was then added and concentrated right at the contacting points of cutting edge and fibers.

2) When fibers breaking was contributed by cutting edge, sliding cutting showed more advantage than orthogonal cutting. The machined surface roughness of sliding cutting is smaller when FOA is $45^{\circ}$ and $90^{\circ}$. When FOA is $0^{\circ}$, however, fibers and matrix were peeled off instead of cut by cutting edge. In this situation, the machined surface roughness of sliding cutting became worse than that of orthogonal cutting.

2) There was barely subsurface damage for both sliding and orthogonal cutting when FOA is $0^{\circ}$. Fibers irregularly breaking and cracks occurred and got worse in orthogonal cutting as FOA increased from $45^{\circ}$ to $90^{\circ}$. After sliding cutting, however, subsurface damages remained lower than orthogonal cutting. Lateral fibers deformation was found in sliding cutting. Though the deformation was undesirable, it is possible controlled and reduced by adjusting the angle between sliding cutting velocity and primary motion.

3) Compared to orthogonal cutting, the cutting force of sliding cutting was reduced $74 \%$ at most. The thrust force, however, was not changed in a large scale since the interaction of flank face and machined surface remained the same in the two cutting mode. Lateral force signals were only detected in sliding cutting.

4) Sliding cutting showed good wear resistance property than orthogonal cutting. The sliding cutting edge rounding radius, an indicator of tool wear, was measured smaller than that of orthogonal cutting after experiencing even a longer cutting distance. 
The FOAs selected in this study were $0^{\circ}, 45^{\circ}$ and $90^{\circ}$. All of them were not larger than $90^{\circ}$. The larger FOA, $135^{\circ}$ for example, was not involved in the sliding cutting experiment. The reason is when FOA gets larger, fibers breaking will not be contributed by cutting edge. Most of the fibers are bended and then broken by rake face. The preferable cutting strategy for the FOAs that larger than $90^{\circ}$ is proposed and defined as transverse cutting by the authors, which will be presented in another paper. Moreover, the temperature was also not considered in the sliding cutting experiments. It will be discussed as a special topic in future work.

\section{Declarations}

\section{Acknowledgment}

This work was supported by Natural Science Foundation of Liaoning Province (Grant number 2021-BS194).

A. Funding: This work was supported by Natural Science Foundation of Liaoning Province (Grant number 2021-BS-194).

B. Conflicts of interest: The authors report no declarations of interest.

C. Availability of data and material: The datasets generated during and/or analyzed during the current study are available from the corresponding author on reasonable request.

D. Code availability: Not applicable.

E. Ethics approval: Not applicable.

F. Consent to participate: Not applicable.

G. Consent for publication: All authors agree to be published in The International Journal of Advanced Manufacturing Technology.

\section{References}

1. Zhang X, Chen Y, Hu J (2018) Recent advances in the development of aerospace materials. Prog Aerosp Sci 97:22-34

2. Wang $X$, Zhang $L$ (2003) An experimental investigation into the orthogonal cutting of unidirectional fibre reinforced plastics. Int J Mach Tools Manuf 43(10):1015-1022

3. An Q, Cai C, Cai X et al (2019) Experimental investigation on the cutting mechanism and surface generation in orthogonal cutting of UD-CFRP laminates. Compos Struct 230:111441

4. Calzada K, Kapoor S, Devor R et al (2012) Modeling and interpretation of fiber orientation-based failure mechanisms in machining of carbon fiber-reinforced polymer composites. J Manuf Process 14(2):141-149 
5. Li C, Zhao Y, Qiu X et al (2022) Interface mechanical damage mechanism in machining carbon fiberreinforced plastic/Ti stacks based on a three-dimensional microscopic oblique cutting model. Compos Struct 279:114737

6. Li H, Wang J, Wu C et al (2020) Damage behaviors of unidirectional CFRP in orthogonal cutting: A comparison between single- and multiple-pass strategies. Compos Part B: Eng 185:107774

7. Usui S, Wadell J, Marusich T (2014) Finite element modeling of carbon fiber composite orthogonal cutting and drilling. Procedia Cirp 14:211-216

8. Zenia S, Ben A, Nouari M et al (2015) Numerical analysis of the interaction between the cutting forces, induced cutting damage, and machining parameters of CFRP composites. Int J Adv Manuf Technol 78(1-4):465-480

9. Hassouna A, Mzali S, Zemzemi F et al (2020) Orthogonal cutting of UD-CFRP using multiscale analysis: Finite element modeling.Journal of Composite Materials, :808734360

10. Liu Y, Li Q, Qi Z et al (2021) Scale-span modelling of dynamic progressive failure in drilling CFRPs using a tapered drill-reamer. Compos Struct 278:114710

11. Xu J, Mansori M (2016) Cutting modeling of hybrid CFRP/Ti composite with induced damage analysis. Materials 9(1):22

12. Shen Y, Yang T, Liu C et al (2021) Cutting force modeling in orthogonal cutting of UD-CFRP considering the variable thickness of uncut material. Int J Adv Manuf Technol 114:1623-1634

13. Su Y (2019) Effect of the cutting speed on the cutting mechanism in machining CFRP. Compos Struct 220:662-676

14. Fu Q, Wu S, Li C et al (2022) Delamination and chip breaking mechanism of orthogonal cutting CFRP/Ti6Al4V composite. J Manuf Process 73:183-196

15. Meng Q, Zhang K, Cheng H et al (2014) An analytical method for predicting the fluctuation of thrust force during drilling of unidirectional carbon fiber reinforced plastics. J Compos Mater 49:699-711

16. Pereszlai C, Geier N (2020) Comparative analysis of wobble milling, helical milling and conventional drilling of CFRPs. Int J Adv Manuf Technol 106(9-10):3913-3930

17. Tashiro T, Fujiwara J, Asahi N (2016) Cutting characteristics in end-milling of CFRP with diamondcoated herringbone tool. Int J Automa-tion Technol 10(3):356-363

18. Jia Z, Fu R, Niu B et al (2016) Novel drill structure for damage reduction in drilling CFRP composites. Int J Mach Tools Manuf 110:55-65

19. Yu Z, Li C, Kurniawan R et al (2019) Drill bit with a helical groove edge for clean drilling of carbon fiber-reinforced plastic. J Mater Process Technol 274:116291

20. Qiu X, Li P, Li C et al (2019) New compound drill bit for damage reduction in drilling CFRP. Int J Precision Eng Manufacturing-Green Technol 6:75-87

21. Su F, Zheng L, Sun F et al (2018) Novel drill bit based on the step-control scheme for reducing the CFRP delamination. J Mater Process Technol 262:157-167 
22. Jia Z, Zhang C, Wang $F$ et al (2020) An investigation of the effects of step drill geometry on drilling induced delamination and burr of Ti/CFRP stacks. Compos Struct 235:111786

23. Astakhov V (2010) Geometry of single-point turning tools and drills. Springer

24. Meng Q, Cai J, Cheng $\mathrm{H}$ et al (2020) Investigation of CFRP cutting mechanism variation and the induced effects on cutting response and damage distribution. Int J Adv Manuf Technol 106:28932907

25. Zhang C, Chen L, Xia J et al (2019) Effects of blade sliding cutting angle and stem level on cutting energy of rice stems. Int J Agricultural Biol Eng 12(6):75-81

26. Hassouna A, Mzali S, Zemzemi F et al (2020) Orthogonal cutting of UD-CFRP using multiscale analysis: Finite element modeling.Journal of Composite Materials. :808734360

27. Li C, Zhao Y, Qiu X et al (2022) Interface mechanical damage mechanism in machining carbon fiberreinforced plastic/Ti stacks based on a three-dimensional microscopic oblique cutting model. Compos Struct 279:114737

28. Wang H, Pei Z, Cong W (2020) A feeding-directional cutting force model for end surface grinding of CFRP composites using rotary ultrasonic machining with elliptical ultrasonic vibration. Int $\mathrm{J}$ Mach Tools Manuf 152:103540

\section{Figures}

\section{Figure 1}

Schematic of typical CFRP cutting

\section{Figure 2}

Sliding cutting

\section{Figure 3}

The forces between cutting edge and fiber during sliding cutting 
Cutting tools and test-pieces in experiments

\section{Figure 5}

Experimental set up and configuration

\section{Figure 6}

Comparison of machined surface and its roughness

\section{Figure 7}

Subsurface of orthogonal and sliding cutting when $\mathrm{FOA}=0^{\circ}$

\section{Figure 8}

Subsurface of orthogonal and sliding cutting when FOA $=45^{\circ}$

\section{Figure 9}

Subsurface of orthogonal and sliding cutting when FOA $=90^{\circ}$

\section{Figure 10}

Forces of sliding and orthogonal cutting 
Measurement and comparison of tool wear

Page 15/15 